\title{
Infrared Synchrotron Radiation: from Condensed Matter to Biology Researches
}

\author{
A. Marcelli and C. Iliescu \\ INFN, Laboratori Nazionali di Frascati, P.O. Box. 13, 00044 Frascati, Italy
}

\begin{abstract}
Infrared spectroscopy is probably the oldest spectroscopic method applied to investigate materials and chemico-physical phenomena. Nowadays, infrared spectroscopy represents the characterization technique most applied in the industry and in many technological processes. In the last decades a significant progress has been achieved in the use of the intense and brilliant infrared emission from electron storage rings previously used only as VUV and $\mathrm{X}$-ray sources. In the infrared range the low energy of the electron beam does not affect the synchrotron radiation spectral distribution, while high current will make storage rings the most brilliant infrared sources to be used for infrared spectroscopy and micro-spectroscopy. Infrared micro-spectroscopy is a unique technique that combines microscopy and spectroscopy for purposes of micro-analysis. Spatial resolution, within a microscopic field of view, is the goal of the modern infrared micro-spectroscopy applied to condensed matter physics, materials science, biophysics, and now to medicine. Although limited in spatial resolution, infrared is able to resolve chemistry using the contrast of the absorption lines. Fourier transform-infrared micro-spectroscopy using synchrotron radiation is now able to collect data with $2-4 \mathrm{~cm}^{-1}$ resolution on the scale of $10-100$ seconds up to an area of a few microns opening a new scenario: infrared spectroscopy of entire cells and tissue. Moreover, distributions of functional groups such as proteins, lipids, and nucleic acids can be achieved inside a single living cell with a spatial resolution of a few microns.
\end{abstract}

PACS numbers: 78.30.-j, 87.64.-t, 87.57.-s, 41.60.Ap, 87.14.Ee

\section{Introduction}

Observations and studies of the interaction of electromagnetic radiation with matter have provided us with much of our knowledge about the intrinsic nature, the geometric and electronic structure of the world surrounding us. Today, spec- 
troscopic methods and photon-scattering experiments of a wide-ranging variety are indispensable tools for basic and applied research in physics, chemistry, and biology. Among different spectroscopical techniques performed to investigate any kind of materials and chemico-physical phenomena, infrared spectroscopy is probably the oldest one [1]. Infrared (IR) light was discovered in 1800 by Sir William Herschel, a British astronomer. Since its discovery, IR spectroscopy has always been a fertile research field for physicists. Chemists discovered IR spectroscopy in the $60 \mathrm{~s}$ and began to investigate very complex molecular structures. Lately biologists adopted IR spectroscopy and many biomolecules have been investigated with IR spectroscopy. The 70s witnessed a revolution that has continued ever since, as Fourier transform (FT) spectrometers became available to a wider scientific community. Among many, IR spectroscopy have important advantages, compared to other techniques, the most important ones are the use of non-ionising radiation and the almost negligible damage to samples under analysis. As a consequence, FT-infrared spectroscopy (FTIR) not only represents one of the most powerful investigation method in science but the characterization technique most applied in the industry and in many technological processes. In the last years, the construction of the new dedicated IR apparatus at synchrotron radiation facilities all over the world is going to modify the established scenario [2]. Already today IR free electron laser (FEL) sources are in operation [3-5] and new sources planned to cover also the X-ray range are under design both in Europe and in USA. We will not mention nor discuss in the next the specific properties and the challenging scientific opportunities provided by FEL's, the most brilliant radiation sources now available, but we will focus only on researches developed using synchrotron radiation (SR).

As is well known, electrons submitted to acceleration field are emitting electromagnetic waves. In circular high energy storage rings with electron energies up to several giga electron volt $(\mathrm{GeV})$ the magnetic field in the bending magnets is inducing a very strong centripetal acceleration to the highly relativistic electrons. The acceleration induces electromagnetic wave emission up to several hundreds of $\mathrm{keV}$. The basic properties of SR consist in:

(a) a broadband extending from X-ray to the far-infrared (FIR, i.e. $10<\omega<$ $300 \mathrm{~cm}^{-1}$ ),

(b) high photon flux compared with conventional sources (e.g., blackbody source for IR),

(c) highly collimated beam (in the IR range up to hundreds of mrad),

(d) small source size,

(e) highly polarized radiation (in the plane of the ring), and

(f) pulsed source useful for time dependent experiments.

The high photon flux combined with the highly collimated beam leads to a high brilliance compared with thermal sources such as those typically used in IR. 
Brilliance (photons $/ \mathrm{mm}^{2} / \mathrm{mrad}^{2} / 0.1 \% \mathrm{bw}$ ) is crucial for microscopy where one is trying to illuminate an area as small as possible with much light as possible. The advantage of IRSR over a conventional source when used to illuminate a diameter sample area of a micrometer size can lead up to several orders of magnitude higher intensity, particularly in the mid-infrared spectral range. Finally, the last advantage of SR is due to the fact that when samples are measured at room temperature, the main noise is the thermal background. In the IR spectral region SR puts the signal well above this background.

The use of electron storage rings as dedicated light source in the infrared spectral range has attracted a lot interest since the last three decades. Stevenson et al. first discussed the SR as an infrared source [6]. Today, high stability and the largest tunability are available to scientists working in the IR region, up to far IR wavelengths with unprecedent brilliance at SR facilities [7]. The results achieved are astonishing, applications are growing fast all over the world and additional facilities have been commissioned or are going to be installed at storage rings in Europe, Japan, and USA [8-10]. Also in Italy a dedicated IRSR facility (SINBAD) is going to be operational. Synchrotron INfrared Beamline at DA $\Phi$ E (SINBAD) has been installed on DA $\Phi N E$, the new collider of the Laboratori Nazionali di Frascati (LNF) designed to work at $0.51 \mathrm{GeV}$ with a beam current $>1 \mathrm{~A}$, and designed to work at wavelengths up to $5000 \mu \mathrm{m}$. Because in the IR range the low energy of the electron beam $(E>0.51 \mathrm{GeV})$ does not affect the SR spectral distribution, while the IR emission is simply proportional to the current, SINBAD promises to be one of the most brilliant IR sources in the world to be used for IR spectroscopy and micro-spectroscopy [11].

\section{Experimental details}

IR micro-spectroscopy is a unique technique that combines microscopy and spectroscopy for purposes of micro-analysis. Although limited in spatial resolution, when compared to the resolution achievable with X-ray, IR is able to resolve chemistry using the contrast method. IR-microscopy is a micro-analytical and imaging technique, which achieves contrast via the intra-molecular vibrational modes. The method is the same used in X-ray microscopy where contrast is achieved recording spectra before and after the absorption edges of an element contained in the specimen. While in light microscopy, image contrast is achieved with stains or fluorescent materials, with IR the use of chemical reagents or stains is not necessary. Image contrast is simply produced from intrinsic IR absorption bands. Indeed, FTIR micro-spectroscopy allows spatially resolved local chemical analysis in situ from small portions of the microscopic field. Instrumentation capable of providing excellent spatial resolution was initially developed for materials and forensic sciences while biological science and medicine come later. The spectrum of a single red blood cell with and without carbon monoxide was reported only 
in 1988 using a microscope connected to a conventional FTIR spectrometer [12]. Thanks to the unique properties of IRSR such as the great brilliance, the lack of thermal noise, the extreme collimation, the polarization, and the spatial resolution (near the diffraction limit), experiments may be now performed with a high signal to noise $\mathrm{S} / \mathrm{N}$ ratio and a resolution which are not reachable by any conventional source. FTIR micro-spectroscopy using SR is now able to collect scans with $2-4 \mathrm{~cm}^{-1}$ of resolution on the scale of $10-100 \mathrm{~s}$ up to area of $3 \times 3 \mu \mathrm{m}[13,14]$. An infrared map is made by scanning the sample in a raster fashion, recording interferograms at each point, and then constructing images at selected wavelengths afterwards. In such an approach it is highly desirable to achieve a spatial resolution as high as possible, although diffraction will provide the ultimate limit. This diffraction-limited resolution cannot be reached when the microscope is equipped with a conventional thermal source due to the lack of energy at the sample position when closing the aperture down (typically below 10 or $15 \mu \mathrm{m}$ ). Only with SR it is possible to approach this limitation. Indeed SR in the IR range has a spectral luminance (brilliance) of about 1000 times (or even more) greater than globar sources, and therefore offers considerably improved S/N capabilities. Particularly for the biological applications, it is now possible to reach spatial resolution at the level of individual cells. Cells vary in shape and size from the most simple to the indescribably complex. Human cells may range from a configuration that is about $10-15 \mu \mathrm{m}$ on edge, and nearly cubic in shape, to stratified morphology, up to $60 \mu \mathrm{m}$ in diameter and $5-10 \mu \mathrm{m}$ thick, depending on the organ of origin. In Fig. 1 we shows a photomicrograph acquired through the Nicolet microscope installed at the 13-3 beamline at Daresbury. The image shows the focused SR spot of dimension of $20 \times 20 \mu \mathrm{m}$ used for FTIR analysis. The illuminated area selects almost only one of the cultured cancerous cells placed on a $\mathrm{CaF}_{2}$ substrate [15]. However, a couple of disadvantages are associated to the microscope methodology: the first is represented by the use of thin samples. In such a way it is not easy to investigate all living subjects. The second is represented by the complex task to identify all the individual contributions of the absorbing molecules present in the IR path and recorded in a single spectrum.

IR spectroscopy of entire cells and tissue represents today a new challenging research that still requires a big effort to achieve the reliability required to detect (e.g., for diagnosing and/or prognosing) various diseases and monitor various disease processes by following the transformation from the healthy state to the diseased state [16]. Because IR spectroscopy can detect and monitor characteristic changes in molecular composition and structure associated to the transformation from the normal to the cancerous state the first reports in the 90 's claimed the capability to detect cancer by comparing IR spectra of healthy and diseased samples [17-19]. Actually FTIR may reveal features characteristic of malignancy that are not yet evident to the pathologist and hence in the near future form the basis for sensitive diagnosis. Recently, using infrared synchrotron radiation it has been 


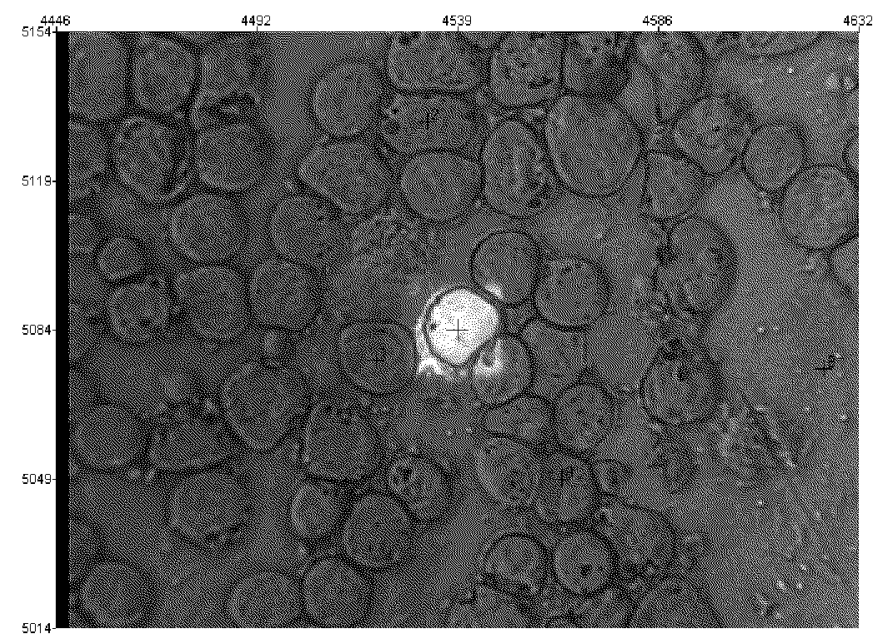

Fig. 1. Photomicrograph acquired through a Nicolet microscope, of cultured cancerous cells placed on a $\mathrm{CaF}_{2}$ substrate. The observed illuminated area by the synchrotron radiation IR spot has dimension of about $20 \times 20 \mu \mathrm{m}$.

possible to map the distributions of functional groups such as proteins, lipids, and nucleic acids inside a single living cell with a spatial resolution of a few microns [20]. Observed spectral changes include all types of lesions induced in that cell as well as cellular responses to external and internal stresses. Spectral changes combined with other analytical tools may provide a fundamental understanding of the key molecular mechanisms induced in response to stresses created by low-doses of chemicals [21].

\section{Results}

IR investigation of biological molecules, whether in model systems in vitro, tissue components in situ, or intact organisms such as bacteria, has become a recognized analytical method over the last few decades. Determination of the structure of a biomolecule is probably the most critical step toward reaching an understanding of its mechanism of action. This is the case when the molecule is a protein, nucleic acid, carbohydrate, lipid, or bio-membrane structure. The IR spectrum of a biological system exhibits well-defined absorption bands (characteristic molecular vibrations) that appear at different positions with different relative intensities. Each spectral difference reflects a potential structural difference in the system. Therefore, vibrational spectroscopy allows defining sets of IR marker absorption bands of the various conformations. In Fig. 2 we show a typical spatially resolved IR spectra of a biological specimen. The spectrum that covers a wide spectral range has been recorded with an IR microscope by averaging data from a reasonable number of scans. The arrows indicate in the near-IR and mid-IR ranges 


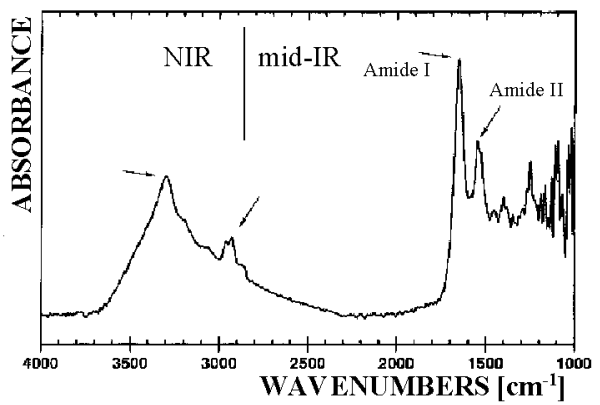

Fig. 2. The typical spatially resolved IR spectra of a biological system recorded with microscope adding several scans. The arrows indicate in the near-IR (NIR) and mid-IR ranges the main IR bands which can be used to recognize and distinguish different samples and materials.

the main IR bands which can be used to recognize and distinguish different samples the amide I profile $\left(1600-1700 \mathrm{~cm}^{-1}\right)$ is useful in identifying a change in the protein conformation.

In the cytoplasm of a cell, the protein concentration is about $100 \mathrm{mM}$. Since they are the most abundant species in cells, their contributions dominate the IR spectra of cells and tissue. Also the average concentration of DNA in cell's nucleus is extremely high and also the highly polymorph DNA can be investigated in the IR-range in order to investigate the cell cycle or to address issues associated with the conformational transitions between the various helical geometries of proteins [16].

Proteins are probably the most important active agents in biochemistry: without them metabolic processes and life would not take place. From a chemical viewpoint, proteins are linear heteropolymers. Most of the recent investigations devoted to proteins concentrate on their catalytic abilities, chemical kinetics, interactions, etc. almost neglecting the overall structure of the protein (which is much larger than the active-site) [22]. The organization of the majority of proteins is such that it folds as relatively small self-contained structures. The folding of a protein involves the search for a single native structure within a large ensemble of conformations that are accessible to the polypeptide chain after its biosynthesis. To solve the problem of protein structure and folding there is still a need for methods to discriminate between the various structures and distinguish between native and non-native structures during the folding reaction [23]. FTIR spectroscopy has emerged as a powerful method for studying the secondary structure of proteins and subtle changes in the protein structure [24]. Infrared absorbance spectra are not subjected to structural averaging as in NMR for flexible states that undergo fast conformational exchange. This means that FTIR can distinguish between native and non-native secondary structures present in partially folded states. Besides the understanding of the complex structure of proteins, it is very important to try 
to detect and maybe to quantify proteins that are contained in individual cells without using chemical reagents or stains.

The p53 protein actively suppresses tumor formation; when it is mutated, the road is open for the development of several forms of cancer. Mutations in p53 or the pathway that directly regulates it have been found in over $80 \%$ of human tumors, so there has been intense research into the p 53 gene and its product. One of p53's functions in the damage response is the activation of genes that initiate apoptosis. Selective removal of severely damaged cells is thought to protect an organism from cancer. The concentration of this protein is very low in normal cells but increases quickly once an oncogene distress signal appeared (DNA damage caused by different factors). p 53 has been shown to arrest the cell cycle in response to DNA damage, thus preventing the replication of damaged DNA. However, the amount of p 53 protein in cells is determined mainly by the rate at which it is degraded, rather than the rate at which it is made. p 53 protein is binding on DNA via the active site that contains a $\mathrm{Zn}$ atom with 4 directly covalent bonds, 3 with $\mathrm{S}$ atoms and 1 to an $\mathrm{N}$ atom. To measure the IR spectrum of $\mathrm{p} 53$, of which we started to calculate the position of the $\mathrm{Zn}_{n}$ atom inside the active site and the changes of the position in mutant or denatured proteins, we designed and built a specific cell for liquids using Cleartran ${ }^{\mathrm{R}}$ windows to be used for FTIR spectroscopy and microscopy experiments.

IR spectra of a very concentrate human p53 (200 $\mu \mathrm{g}$ of pure protein dissolved in $40 \mu \mathrm{l}$ of PBS, $5 \mathrm{mM}$ DTT and $50 \%$ Glycerol), provided by Santa Cruz Biotechnology with a special preparation were measured at the $13-3$ beamline at Daresbury using the IR emission of the Synchrotron Radiation Centre (SRC) storage ring. Spectra of the biological liquids containing the p 53 protein provided by Santa-Cruz Biotech were sampled as a function of time and compared. Because at the 13-3 beamline, the acquisition time with this concentration is about $30 \mathrm{~s}$ per spectrum, with FTIR the dynamics of the denaturation may be investigated. Figure 3 clearly shows the differences among IR transmission spectra of p 53 protein in solution. The first difference (circle dots) obtained subtracting the original stored spectrum with a one stored about $500 \mathrm{~s}$ later shows an abrupt change correlated with the denaturation process during the data acquisition at room temperature (the protein was kept till the experiment at $-20^{\circ} \mathrm{C}$ ). The second curve (square dots) of Fig. 3, has been achieved subtracting the second spectrum with one stored about $1000 \mathrm{~s}$ later, and outlines the presence of a second step of the process where two bands are involved. The two components appear in the range of $1900-1600 \mathrm{~cm}^{-1}$ and probably identify changes in the protein conformation [25].

In addition to experiments in solution, an IR-microscope allows for spectral acquisition at focal spots on a thin tissue section. Certain assigned spectral features found in the collection of pixel or point spectra of a tissue "map" can then be compared to reveal the distribution of tissue constituents. An increasing range of tissue types is being studied [26], including uterine endometrium, exfoli- 


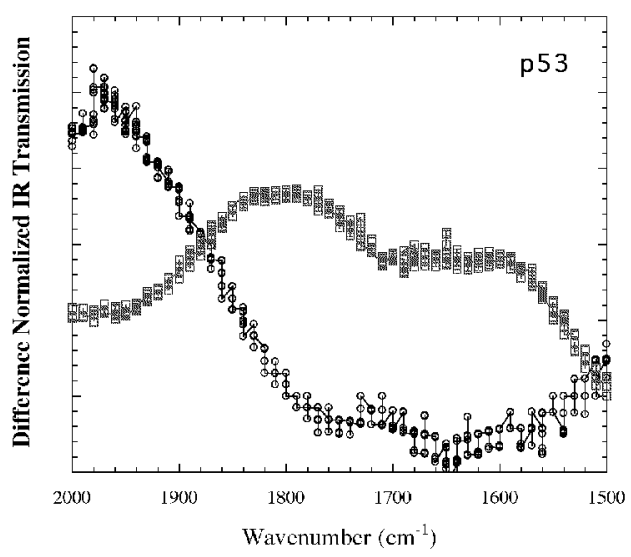

Fig. 3. Differences among IR transmission spectra of p53 protein in solution (see text). The first curve (circle dots) shows an abrupt change of the spectrum profile in the initial phase (about $300 \mathrm{~s}$ ) of the quick denaturation process during the acquisition at room temperature. The second curve (square dots) is related to the second step of the process where two components are involved.

ated cervical cells, bone, dermal, intestine, liver, as well as histological brain tissue for studies about the Alzheimer disease. Scientists have started to investigate the characteristic differences between the spectra of human grey matter, white matter and chronic lesions to characterize Alzheimer's disease and multiple sclerosis. Alzheimer's disease is currently incurable, and due to its nature, as many neurological disorders, is difficult to diagnose. There is some evidence that early therapeutic intervention may at least retard the progress of this debilitating disease. To this end, non-subjective methods for the early diagnosis are required. In Canada scientists are establishing an infrared spectroscopic data bank for white matter, grey matter, and brain matter affected by multiple sclerosis or Alzheimer's disease. They have been able to identify characteristic differences between the spectra of human grey matter, white matter, and chronic lesions, based on differences in lipid, protein, and water content of the various tissues. The presence of clear chemical differences and the presence of several peaks in order to distinguish white from grey matter in the spectra was early established with IR experiments obtained by spatially resolved in situ probing of a cerebrum section of normal mouse [27]. More recently one investigation addresses the role of the organization of proteins in amyloid plaques [28]. In individuals with Alzheimer's disease preponderance of $\beta$-sheet structure is observed (amide I at $1630-1634 \mathrm{~cm}^{-1}$ ) while in the surrounding tissue the proteins present mostly an unordered a-helical conformation (amide I - 1652-1654 $\mathrm{cm}^{-1}$ ).

IR micro-spectroscopy is capable, for instance, of discriminating between histological tissue samples containing various amounts of cancerous material. Many 
cell types are likely to be present within tumour tissue, depending on its origin, such as malignant invasive cells or epithelial cells. It has been shown that the IR-spectra display a clear differentiation between cells within the normal epithelium and those with the tumour $[29,30]$. It can detect and monitor characteristic changes in molecular composition and structure that accompany the transformation from the normal to the cancerous state. Analysis of spectra can help a clinician to localize a tumour and provide a probability of whether or not it is cancerous. The spectroscopic fingerprints of many forms of cancer (cervical, colon, skin, breast, brain, oral, prostate, thyroid, leukemia) and a number of cardiovascular disorders can be evaluated. The IR-microscopy reveals to be a powerful diagnostic tool and should allow to achieve a better insight on the mechanisms responsible for the loss of cell differentiation within the tumour as cells begin to divide rapidly and invade the surrounding tissue. The challenge is to develop non- or minimally-invasive methods to detect these conditions in living patients, in order to provide an early diagnosis of this debilitating disease.

The main advantages of IR analysis relate to the simplicity of the measurement, and the high information content of the spectra. The foundation of clinical chemistry is the fact that certain diseases give rise to characteristic changes in the chemistry of serum, urine, or other fluids. Several key analytes may be estimated simultaneously from a spectrum. For example, a level of proteins such as urine creatinine, urea, etc. may all be evaluated from the near-infrared spectrum in less than a minute. This spectroscopy offers a new window on the analysis of the biofluids, its speed, and the fact that no reagents are required, makes IR clinical analysis very attractive [31, 32].

\section{Conclusions}

Advances in IR spectroscopy during the last few years are certainly due to the availability of SR sources, however improvements in measuring techniques and refinements in data processing cannot be neglected. The main advantage of IR spectroscopy for the study of biomolecules is that high quality spectra can be obtained with a variety of environments such as aqueous solution, organic solvents, and even in the presence of other biomolecules. Moreover, IR-microscopy can be used to probe intact living cells providing a composite view of all of the molecular responses and the ability to monitor the responses over time in the same cell. Actually, another important feature of IR technique using SR is the potential for slow and fast kinetic studies. FTIR spectroscopy, able to investigate samples in crystalline, polycrystalline, amorphous, and solutions states, in the next years may be able to clarify many apparently contradictory data obtained in solid state by X-ray crystal diffraction and in solution by NMR. IR spectroscopy has the particular advantage over NMR that it does not require any solvents. Moreover, the interpretation of the IR experimental data is not complicated by time scale 
averaging of anisotropic motions, since the time scale of IR spectroscopy is very fast (about $10^{-12} \mathrm{~s}$ ) compared to the ESR and NMR time scale $\left(10^{-5}-10^{-8} \mathrm{~s}\right.$ ).

About the "cancer detection" via IR spectroscopy, we have to underline that, although FTIR represents an invaluable tool able to provide information on biochemistry, cellular composition, cell components, metabolic processes, presence of disease and even more, at the present day the capability to interpret independently cancerous vs. normal tissues data still has not been achieved. Nevertheless, changes in IR spectra can be explained unambiguously in terms of compositional change so that IR data contain medical information that will be really used as a tool in pathology in the near future. As a consequence, in medicine the primary objective of IR micro-spectroscopy will monitor various disease processes by following the transformation from the healthy state to the diseased state. The development of independent spectroscopic and imaging methods capable of detecting (diagnosing and/or prognosing) such transformations at an early stage is the task of the next years.

We had no space here to show the impact that IR may have on industry connected with biological researches. However, each year the cosmetic industry reaps billions of dollars from sales of products designed to enhance skin and hair, tissues composed primarily of keratin, a tough, insoluble protein. Hair keratin undergoes oxidation by both photochemical and chemical means resulting in decreased tensile strength, colour changes and easily abraded cuticle. Because of products to cleanse, moisturize or condition skin and hair abound, in order to compete, industry has to promote researches devoted to the understanding of the composition and structure of skin and hair and on the nature of the interactions between these substrates and the products themselves. IR spectroscopy already plays an important role in these industrial oriented researches.

\section{Acknowledgment}

We gratefully acknowledged M. Tobin and M.G. Furfaro for the invaluable support during the experimental runs at Daresbury. It is a pleasure to acknowledge E. Righi, G. Trenta, and C. Catena for many stimulating discussions.

\section{References}

[1] E.F. Nichols, Phys. Rev. B 1, 1 (1993).

[2] J.A. Reffner, P.A. Martoglio, G.P. Williams, Rev. Sci. Instrum. 66, 1298 (1995).

[3] M. Poole, in: Synchrotron Radiation and Free Electron Lasers, Ed. S. Turner, CERN 90-03, Geneve 1990, p. 306.

[4] J.-M. Ortega, in: Accelerator-based Infrared Sources and Applications, Eds. G.P. Williams, P. Dumas, Proc. SPIE 3153, 2 (1997).

[5] T.I. Smith, in: Accelerator-based Sources of Infrared and Spectroscopic Applications, Eds. G.L. Carr, P. Dumas, Proc. SPIE 3775, 46 (1999). 
[6] J.R. Stevenson, H. Ellis, R. Bartlett, Appl. Opt. 12, 2884 (1973).

[7] R.P.S. Lobo, J.D. LaVeigne, D.H. Reitze, D.B. Tanner, G.L. Carr, Rev. Sci. Instrum. 70, 2899 (1999).

[8] Infrared Synchrotron Radiation, Eds. P. Calvani, P. Roy, Editori Compositori, Bologna 1998.

[9] Accelerator-based Infrared Sources and Applications, Eds. G.P. Williams, P. Dumas, Proc. SPIE 3153, (1997).

[10] Accelerator-based Sources of Infrared and Spectroscopic Applications, Eds. G.L. Carr, P. Dumas, Proc. SPIE 3775, (1999).

[11] A. Marcelli, A. Nucara, D. Cannavo', E. Burattini, P. Calvani, G. Cinque, C. Mencuccini, S. Lupi, F. Monti, in: Accelerator-based Sources of Infrared and Spectroscopic Applications, Eds. G.L. Carr, P. Dumas, Proc. SPIE 3775, 7 (1999).

[12] A. Dong, R.G. Messerschmidt, J.A. Refnner, Biochem. Biophys. Res. Commun. 156, $752(1988)$

[13] D.L. Wetzel, S.M. LeVine, in: Infrared and Raman Spectroscopy of Biological Materials, Eds. H.-U. Gremlich, Bing Yan, Marcel Dekker, New York 2001, p. 101.

[14] D.L. Wetzel, S.M. LeVine, Science 285, 1224 (1999).

[15] M.G. Furfaro, S. Gaudenzi, C. Mliescu, A. Marcelli, M. Tobin, A. Congiu Castellano, Infrared Study of Cells Doped with Heavy Metals, contribution III-119 at the INFM - Meeting 2001 (June 18-22, Roma), unpublished.

[16] M. Diem, S. Boydston-White, L. Chiriboga, Appl. Spectrosc. 53, 148A (1999).

[17] B. Rigas, S. Morgello, I.S. Goldman, P.T.T. Wong, Proc. Natl. Acad. Sci. USA 87, $8140(1990)$.

[18] D.C. Malins, N.L. Polissar, K. Nishikida, E.H. Holmes, H.S. Gardner, S.J. Gunselman, Cancer 75, 504 (1995).

[19] H. Fabian, M. Jackson, L. Murphy, P.H. Watson, I. Fichtner, H.H. Mantsch, Biospectroscopy 1, 37 (1995).

[20] N. Jamin, P. Dumas, J. Moncuit, W.H. Fridman, J.L. Teillaud, G.L. Carr, G.P. Williams, Proc. Natl. Acad. Sci. USA 95, 4837 (1998).

[21] Hoi-Ying, N. Holman, R. Goth-Goldstein, M.C. Martin, M.L. Russel, W.R. McKinney, Environ. Sci. Technol. 34, 2513 (2000).

[22] W.R. Taylor, A.C.W. May, N.P. Brown, A. Aszodi, Rep. Prog. Phys. 64, 517 (2001).

[23] A. Troullier, D. Reinstädler, Y. Dupont, D. Naumann, V. Forge, Nat. Struct. Biol. $7,78(2000)$.

[24] W.E. Surewicz, H.H. Mantsch, D. Chapman, Biochemistry 32, 389 (1993).

[25] C. Iliescu, A. Marcelli, private communication.

[26] Biomedical Spectroscopy: Vibrational Spectroscopy and Other Novel Techniques, Eds. A. Mahadevan-Jansen, G.J. Puppels, Proc. SPIE 3918, (2000).

[27] D.L. Wetzel, S.M. LeVine, Spectrosc. 8, 40 (1993).

[28] L.-P. Choo, D.L. Wetzel, W.C. Halliday, M. Jackson, S.M. LeVine, H.H. Mantsch, Biophysics J. 71, 1672 (1996). 
[29] L. Chiriboga, P. Xie, H. Yee, D. Zarou, D. Zakim, M. Diem, Cell. Mol. Biol. 44, 219 (1998).

[30] M. Romeo, F. Burden, M. Quinn, B. Wood, D. McNaughton, Cell. Mol. Biol. 44, 179 (1998).

[31] R.A. Meyers, Encyclopedia of Analytical Chemistry: Instrumentation and Application, Wiley, New York 2000, p. 4344.

[32] A. Bittner, H.M. Heise, AIP Conf. Proc. 430, 278 (1998). 